\title{
TU/e EmonONEN

\section{Partial slip boundary conditions for collisional granular flows at flat frictional walls}

\section{Citation for published version (APA):}

Yang, L., Padding, J. T., \& Kuipers, J. A. M. (2017). Partial slip boundary conditions for collisional granular flows at flat frictional walls. AlChE Journal, 63(6), 1853-1871. https://doi.org/10.1002/aic.15534

DOI:

10.1002/aic.15534

Document status and date:

Published: 01/06/2017

\section{Document Version:}

Accepted manuscript including changes made at the peer-review stage

\section{Please check the document version of this publication:}

- A submitted manuscript is the version of the article upon submission and before peer-review. There can be important differences between the submitted version and the official published version of record. People interested in the research are advised to contact the author for the final version of the publication, or visit the $\mathrm{DOI}$ to the publisher's website.

- The final author version and the galley proof are versions of the publication after peer review.

- The final published version features the final layout of the paper including the volume, issue and page numbers.

Link to publication

\section{General rights}

Copyright and moral rights for the publications made accessible in the public portal are retained by the authors and/or other copyright owners and it is a condition of accessing publications that users recognise and abide by the legal requirements associated with these rights.

- Users may download and print one copy of any publication from the public portal for the purpose of private study or research.

- You may not further distribute the material or use it for any profit-making activity or commercial gain

- You may freely distribute the URL identifying the publication in the public portal.

If the publication is distributed under the terms of Article 25fa of the Dutch Copyright Act, indicated by the "Taverne" license above, please follow below link for the End User Agreement:

www.tue.nl/taverne

Take down policy

If you believe that this document breaches copyright please contact us at:

openaccess@tue.nl

providing details and we will investigate your claim. 


\title{
Partial slip boundary conditions for collisional granular flows at flat frictional walls
}

\section{L. (Lei) Yang, J.T. (Johan) Padding*, J.A.M. (Hans) Kuipers}

Department of Chemical Engineering and Chemistry, Eindhoven University of Technology, 5600 MB, Eindhoven, The Netherlands

* Corresponding author. Current address: Process \& Energy department, Delft University of Technology, The Netherlands.J.T.Padding@tudelft.nl

Keywords: Frictional collision, rough particles, kinetic theory of granular flow, partial slip boundary, Two-fluid model

\begin{abstract}
We derive new boundary conditions (BCs) for collisional granular flows of spheres at flat frictional walls. A new theory is proposed for the solids stress tensor, translational and rotational energy dissipation rate per unit area and fluxes of translational and rotational fluctuation energy. In the theory we distinguish between sliding and sticking collisions and include particle rotation. The predictions are compared with literature results obtained from a discrete particle model evaluated at a given ratio of rotational to translational granular temperature. We find that the new theory is in better agreement with the observed stress ratios and heat fluxes than previous kinetic theory predictions. Finally, we carry out two fluid model simulations of a bubbling fluidized bed with the new BCs, and compare the simulation results with those obtained from discrete particle simulations. The comparison reveals that the new BCs are better capable of predicting solids axial velocity profiles, solids distribution near the walls and granular temperatures.
\end{abstract}

\section{Introduction}

The Euler-Euler two fluid model (TFM) is the most popular approach to simulate large scale gassolid flows in various processes involving separation, coating and mixing. The kinetic theory of granular flow can be used to derive the transport equations of mass, momentum and fluctuation energy of the 
solid phase, as well as expressions for bulk transport coefficients such as the bulk and shear viscosity and the transport coefficient for the fluctuation energy. Besides these transport equations and bulk properties, boundary conditions in terms of the primary variables, i.e. pseudo thermal conductivity, need to be specified at the locations of walls. The importance of wall boundary conditions should not be underestimated. An indication of the importance of wall boundary conditions in determining the characteristics of granular flow has been provided by experiments by Hanes and $\operatorname{Inman}^{1}$ and Sommerfeld and Huber ${ }^{2}$. Numerical simulations ${ }^{3-4}$ were also employed to show the important role of wall boundary conditions. In rapid granular flows, the interactions between the solid particles and the wall lead to a rapid succession of almost instantaneous collisions. These collisions cause random fluctuations of the particle velocities, which determine the momentum and fluctuation energy transfer rates to the walls ${ }^{5}$.

Several theoretical studies have incorporated the physics of particle-wall collisions into granular flows. Hui et al. ${ }^{6}$ modeled the wall interaction as an equivalent-roughness coefficient but neglected the shear work performed by the wall due to the slip velocity at the surface. Jenkins and Richman ${ }^{7}$ developed boundary conditions for smooth circular disk flows in the neighborhood of a boundary composed of semicircular hemispheres attached to a flat wall. The BCs in these approaches requested a strong restrictive condition on the particle density at the wall. The boundary may supply fluctuation energy to the flow due to slip.

Johnson and Jackson ${ }^{8}$ introduced heuristic boundary conditions by assuming that some particles collide and some particles slide relative to the wall. They characterized the momentum and energy transfer due to collisions between particles and wall by a "specularity coefficient" where its value depends on the roughness of the wall and varies between zero for perfectly specular collisions and unity for perfectly diffuse collisions. Johnson and Jackson BCs are widely used due to their simplicity and flexibility $^{5}{ }^{9-10}$. However, this coefficient has to be adjusted in simulations under different operation conditions (i.e. superficial gas velocity) to achieve good agreement with the experimental data ${ }^{9,11}$. Benyahia et al. ${ }^{3}$ reported that better agreement with experimental data was achieved by using a small specularity coefficient. Sinclair and Jackson ${ }^{12}$ presented a model based on the work of Hui et al. ${ }^{6}$ for the fully-developed flow of gas-solid in a vertical pipe that treated the particle phase as a rapid granular 
flow. This model has been used by many others for the modelling of gas-solid flows, e.g. by Nieuwland et al. ${ }^{13}$, Lu and Gidaspow ${ }^{14}$, and Verma et al. ${ }^{15}$. It is important to note that the original Johnson \& Jackson, and Sinclair \& Jackson boundary conditions do not account for particle-wall frictional effects. In contrast, the more recent boundary conditions of Jenkins ${ }^{16}$, Jenkins and Louge ${ }^{17}$, and Konan et al. ${ }^{18}$ extended different approaches, originally developed for smooth walls.

Jenkins $^{16}$ developed a theory for rapid granular flows interacting with a flat, frictional wall, incorporating Coulomb friction and both tangential and normal restitutions. Based on a simple velocity distribution function, he derived analytical expressions for the shear stress and the flux of fluctuation energy at the wall in two limits, which were "small friction/all sliding” and "large friction/no sliding”, respectively. Jenkins and Louge ${ }^{17}$ refined the calculations of the flux of fluctuation energy and extended the expression by connecting the two limits. The main drawback of these derivations is that the feedback effect of the boundary on the distribution function of the incoming particles is neglected ${ }^{19}$.

Schneiderbauer et al. ${ }^{20}$ developed BCs which include sliding and non-sliding conditions in a single expression. This model was proven to be more accurate in predicting the flux of fluctuation energy in comparison with the other models ${ }^{16-17}$. Furthermore, the general form of this model accounts for a nonsteady state condition of moving walls by accounting for the compression and expansion of the granular flow. However, they neglected the contribution of the rotational energy, which cannot be ignored for granular flows near walls due to the dissipation associated with the inelasticity of the particle-wall collisions. Zhao et al. ${ }^{21}$ derived a set of BCs for collisional granular flows of frictional and rotational particles at flat walls with both translational and rotational granular temperature. The new model could agree better with the literature data $a^{5}$ than the previous models ${ }^{16,20,22}$ for a system artificially set a constant ratio of rotational to translational granular temperature. They pointed out that the particle rotational velocity has a significant impact on both the shear stress and the flux of fluctuation energy. Their expressions for the flux of pseudothermal energy to the wall only considered the rate of dissipation per unit area due to sticking (rolling, non-slipping) particle-wall collisions, and neglected the contribution of particle slip.

Soleimani et al. ${ }^{23}$ derived a new boundary condition in the framework of kinetic theory for frictional walls by using the idea of virtual wall angle of Sommerfeld and Huber ${ }^{2}$. They generalized the above 
wall collision model to be compatible with the two-fluid model. Agreement with experimental data was sought by adjusting the coefficient of wall-friction or by applying the wall roughness model for different degrees of wall-roughness. Fairly good agreement with experimental data could be obtained using their wall-roughness model.

Fede et al. ${ }^{24}$ investigated the effect of the particle-wall BCs in a 3D dense pressurized fluidized bed both numerically and experimentally. They used rough wall boundary conditions from Johnson and Jackson $^{8}$ and smooth wall boundary conditions from Sakiz and Simonin ${ }^{25}$ for the solid phase. They found that rough wall boundary conditions were in better agreement with the experimental data obtained from Positron Emission Particle Tracking in the near-wall region than flat smooth wall boundary conditions. However, the specularity coefficient in Johnson and Jackson's model ${ }^{8}$ cannot be directly measured.

In this work, we propose a theory for the momentum and energy transfer between a flow of granular particles and a frictional wall. The theory describes the collisions between frictional particles and flat walls physically, and adopts both rotational and translational granular temperature. Our analytical expressions for the flux of translational and rotational fluctuation energy account for both the particle slip at the wall and for sticking (rolling) particle-wall collisions. We compare our theory for steady state flows with the data from Louge ${ }^{5}$, using Jenkins and Zhang's theory ${ }^{26}$ for the prediction of the ratio of rotational to translational granular temperature. Alternatively, we can obtain this ratio directly from our in-house two fluid model code which incorporates the kinetic theory of granular flows for rough spheres by Yang et al. ${ }^{27}$. Based on the theory, we derive new BCs for particle slip velocity, and the flux of translational and rotational fluctuation energy in the framework of our kinetic theory. Finally, simulations of a bubbling pseudo-2D fluidized bed using a two fluid model with the new BCs, a two fluid model with Sinclair and Jackson's BCs ${ }^{12}$, and a discrete particle model have been performed to investigate the performance of our new BCs.

\section{Theory}

\section{Particle-wall collisions}


The dynamics of the particle-wall collisions are based on Louge ${ }^{5}$ and Jenkins ${ }^{16}$. Three coefficients are introduced to characterize the dissipative collisions $(e, \beta, \mu)$. It is important to note that the tangential coefficient of restitution applies to the tangential velocity of the contact point and not of the center of mass of the particle.

As shown in Figure 1, the particle has translational velocity $\mathbf{c}$ and angular velocity $\boldsymbol{\omega}$ before the collision; the corresponding post-collision velocities are denoted by primes. Each spherical particles has a mass $m$, diameter $\sigma$, and moment of inertia $I$ around its center of mass. Then, the velocities before and after a collision are related by,

$$
\begin{gathered}
m\left(\mathbf{c}^{\prime}-\mathbf{c}\right)=\mathbf{J} \\
I\left(\boldsymbol{\omega}^{\prime}-\boldsymbol{\omega}\right)=-\frac{\sigma}{2} \mathbf{n} \times \mathbf{J}
\end{gathered}
$$

where $\mathbf{J}$ is the collisional impulse and $\mathbf{n}$ is the wall normal unit vector (directed from the wall into the granular flow). For a static wall, the relative velocity $\mathbf{G}$ at the contact point is given by

$$
\mathbf{G}=\mathbf{c}-\frac{\sigma}{2} \boldsymbol{\omega} \times \mathbf{n}
$$

When the collision angle $\theta$ between $\mathbf{G}$ and $\mathbf{n}$ is in the range $\pi / 2<\theta<\theta_{c}$, where $\theta_{c}$ is a critical collision angle, a sliding collision occurs. In that case the collisional impulse is

$$
\mathbf{J}=-m(1+e)(\mathbf{G} \cdot \mathbf{n}) \mathbf{n}-m \mu(1+e) \cot \theta(\mathbf{G} \times \mathbf{n}) \times \mathbf{n}
$$

On the other hand, for a sticking collision $\left(\theta_{c}<\theta<\pi\right)$, the collisional impulse is

$$
\mathbf{J}=-m(1+e)(\mathbf{G} \cdot \mathbf{n}) \mathbf{n}+m \frac{q}{1+q}(1+\beta)(\mathbf{G} \times \mathbf{n}) \times \mathbf{n}
$$

where $q=\frac{4 I}{m \sigma^{2}}$. For a homogeneous sphere $I=m \sigma^{2} / 10$ and therefore $q=2 / 5$. From the above two equations, the critical angle $\theta_{c}$ can be determined as: 


$$
\cot \theta_{c}=-\frac{q}{1+q} \frac{1+\beta}{\mu(1+e)} \equiv \mu_{0}
$$

where we have defined the parameter $\mu_{0}$ as the cotangent of the critical angle. Note that $\mu_{0} \leq 0$ because of our choice of coordinates. The total collisional impulse from Eqs. 4 and 5 can be put in one expression as

$$
\mathbf{J}=-m(1+e)(\mathbf{G} \cdot \mathbf{n}) \mathbf{n}+m \mu(1+e)\left\{\begin{array}{cl}
G_{n} \mathbf{t}, & \text { sliding } \\
\mu_{0} G_{t} \mathbf{t}, & \text { sticking }
\end{array}\right.
$$

where the unit tangential vector defined as $\mathbf{t}=-(\mathbf{G} \times \mathbf{n}) \times \mathbf{n} /|\mathbf{G} \times \mathbf{n}|$ is oriented parallel to the wall surface, and $G_{n}=\mathbf{G} \cdot \mathbf{n}$ and $G_{t}=\mathbf{G} \cdot \mathbf{t}$ are projections of the relative velocity on the unit normal and tangential vectors. The corresponding energy changes per collision are respectively $\Delta E_{\text {tran }}$ and $\Delta E_{\text {rot }}$, which are given by

$$
\begin{aligned}
& \Delta E_{\text {tran }}=\frac{1}{2} m\left(c^{\prime 2}-c^{2}\right) \\
& =-\frac{m}{2}\left(1-e^{2}\right) G_{n}^{2}+\frac{m}{2} \mu(1+e)\left\{\begin{array}{c}
2 G_{n} \mathbf{c} \cdot \mathbf{t}+\mu(1+e) G_{n}^{2}, \text { sliding } \\
2 \mu_{0} G_{t} \mathbf{c} \cdot \mathbf{t}+\mu(1+e) \mu_{0}^{2} G_{t}^{2}, \text { sticking }
\end{array}\right. \\
& \Delta E_{\text {rot }}=\frac{1}{2} I\left(\omega^{2}-\omega^{2}\right) \\
& =\frac{m \sigma}{2} \mu(1+e)\left\{\begin{array}{c}
-\boldsymbol{\omega} \cdot(\mathbf{n} \times \mathbf{t}) G_{n}+\frac{m \sigma}{4 I} \mu(1+e) G_{n}{ }^{2}, \text { sliding } \\
-\boldsymbol{\omega} \cdot(\mathbf{n} \times \mathbf{t}) \mu_{0} G_{t}+\frac{m \sigma}{4 I} \mu \mu_{0}{ }^{2}(1+e) G_{t}{ }^{2}, \text { sticking }
\end{array}\right.
\end{aligned}
$$

Here, unlike Schneiderbauer et al. ${ }^{20}$ and Zhao et al. ${ }^{21}$, we keep the projected particle spin $\boldsymbol{\omega} \cdot(\mathbf{n} \times \mathbf{t})$ as it exists, without any restrictive assumption. Working this term out further, the interested reader will find that

$$
\boldsymbol{\omega} \cdot(\mathbf{n} \times \mathbf{t})=(\boldsymbol{\omega} \times \mathbf{n}) \cdot \mathbf{t}=\frac{G_{i}\left(\Omega_{j}+\bar{\omega}_{j}\right)-G_{j}\left(\Omega_{\uparrow}+\bar{\omega}_{i}\right)}{G_{t}}
$$


where $\boldsymbol{\Omega}$ is the peculiar rotational velocity, relative to the locally averaged angular velocity $\overline{\boldsymbol{\omega}}=\langle\boldsymbol{\omega}\rangle$, and $\mathbf{i}$ and $\mathbf{j}$ are two mutually perpendicular but fixed axes parallel to the wall surface (see Figure 1). In our tests and comparisons with DPM we will use a correlation for the dependence of the locally averaged angular velocity $\overline{\boldsymbol{\omega}}$ on the locally averaged particle velocity $\mathbf{u}=\langle\mathbf{c}\rangle$. This correlation is obtained from our discrete particle model (DPM) simulations, which we carried out for a fluidized bed containing particles with friction coefficients $0.1,0.3$, and 1.0 at superficial gas velocities $1.5,2.5,3.0$, 3.5 $\mathrm{U}_{m f}$. In agreement with the work of Schneiderbauer et al. ${ }^{20}$, we found that to good approximation the following expression reads

$$
\bar{\omega}_{t} \approx-\frac{\mu}{\sigma} u_{t \times n}
$$

where the component $t$ can be $i$ or $j$ and $t \times n$ indicates the direction perpendicular to both $t$ and $n$ in a right-handed coordinate system (in other words, $\overline{\boldsymbol{\omega}} \approx-\frac{\mu}{\sigma} \mathbf{u} \times \mathbf{n}$ ). The use of this correlation leads to a coupling of the average particle translational velocity to the generation of rotational fluctuations near the walls in our system.

\section{Probability distribution functions}

A similar approach is used here as in Yang et al. ${ }^{27}$. We define $\mathbf{C}(\mathbf{C}=\mathbf{c}-\mathbf{u})$ and $\left.\boldsymbol{\Omega}(\boldsymbol{\Omega}=\mathbf{(})-\overline{\mathbf{\omega}}\right)$ as the peculiar translational and angular velocities relative to the locally averaged particle velocity $\mathbf{u}$ and locally averaged angular velocity $\overline{\boldsymbol{\omega}}$. The translational and rotational granular temperatures are a measure for the mean square peculiar velocities. More precisely they are given by $\Theta_{t} \equiv\left\langle C^{2}\right\rangle / 3$ and $\Theta_{r} \equiv I\left\langle\Omega^{2}\right\rangle / 3 m$

During particle-wall collision, the slip velocities of the particle at the wall are not necessarily zero. Typically, these quantities can be determined as part of a solution to a boundary value problem. Furthermore, the boundary conditions could be solved by considering the collisional exchange of linear 
momentum, angular momentum, and energy loss at the wall. A particle would collide with the wall only when the particle travels towards the wall, $\mathbf{G} \cdot \mathbf{n} \leq 0$. During a collision, the particle property changes from $\psi$ to $\psi^{\prime}$. As is reported in Jenkins ${ }^{16}$, the collisional rate of change Coll $(\psi)$ of $\psi$ per unit area of the wall is the integral of the change in $\psi$ in a single collision over all collisions per unit area,

$$
\operatorname{Coll}(\psi)=-g_{0} \iint_{\mathbf{G} \cdot \mathbf{n} \leq 0}\left(\psi^{\prime}-\psi\right) f(\mathbf{c}, \boldsymbol{\omega}) \mathbf{c} \cdot \mathbf{n} \mathrm{d} \mathbf{c d} \boldsymbol{\omega}
$$

where $g_{0}$ is a factor accounting for the presence of the wall and affecting the spatial distribution of the particles and $f(\mathbf{c}, \boldsymbol{\omega})$ is the probability distribution function to find a particle with translational velocity $\mathbf{c}$ and angular velocity $\boldsymbol{\omega}$ near the wall. If the average translational velocity $\mathbf{u}$ and average rotational velocity $\overline{\boldsymbol{\omega}}$ are already known, we only need to make an assumption for the distribution of peculiar translational and angular velocities in the near wall region. As usual, we will assume this distribution be represented by a two-temperature Maxwellian distribution ${ }^{28}$ :

$$
f(\mathbf{C}, \boldsymbol{\Omega})=n\left(\frac{1}{2 \pi \Theta_{t}}\right)^{3 / 2}\left(\frac{I}{2 \pi m \Theta_{r}}\right)^{3 / 2} \exp \left[-\frac{1}{2}\left(\frac{C^{2}}{\Theta_{t}}+\frac{I \Omega^{2}}{m \Theta_{r}}\right)\right]
$$

where $n$ is the particle number density. Following Schneiderbauer et al. ${ }^{20}$, this distribution function can be interpreted as $f=n f_{n} f_{t}$, with uncorrelated components $f_{n}\left(\mathbf{c}_{n}, \boldsymbol{\omega}_{n}\right)$ and $f_{t}\left(\mathbf{c}_{t}, \boldsymbol{\omega}_{t}\right)$ normal and tangential to the wall. The normal component of the particle velocity is given by

$$
f_{n}\left(c_{n}, \Omega_{n}\right)=\left(\frac{1}{2 \pi \Theta_{t}}\right)^{1 / 2}\left(\frac{I}{2 \pi m \Theta_{r}}\right)^{1 / 2} \exp \left[-\frac{1}{2}\left(\frac{\left(c_{n}-u_{n}\right)^{2}}{\Theta_{t}}+\frac{I \Omega_{n}^{2}}{m \Theta_{r}}\right)\right]
$$

It has to be pointed out that the average normal particle velocity $u_{n}$ is not zero near a moving wall. However, in this work we will consider static walls, common for fluidized beds, in which case we can already set $u_{n}=0$. The tangential component of the particle peculiar velocity distribution is given by 


$$
f_{t}\left(\mathbf{c}_{t}, \boldsymbol{\Omega}_{t}\right)=\frac{1}{2 \pi \Theta_{t}} \frac{I}{2 \pi m \Theta_{r}} \exp \left[-\frac{1}{2}\left(\frac{\left(\mathbf{c}_{t}-\mathbf{u}_{t}\right)^{2}}{\Theta_{t}}+\frac{I \Omega_{t}^{2}}{m \Theta_{r}}\right)\right]
$$

We will allow for a slip velocity along the wall, meaning that the average tangential particle velocity $\mathbf{u}_{t}$ is allowed to be non-zero. The next goal is to transform this distribution function to a distribution function for the relative velocity $\mathbf{G}$ at the contact point instead of the center of mass velocity $\mathbf{c}$. This change of variables will greatly simplify the evaluation of collisional integrals (Eq. 11) over quantities which are expressed in terms of $\mathbf{G}$. The most straightforward route is to realize that, according to Eq. 3, the components of $\mathbf{G}$ are linear combinations of components of $\mathbf{C}$ and $\boldsymbol{\Omega}$. Any linear combination of Gaussian random variables is also a random Gaussian variable, with a variance that can simply be obtained by careful adding of original variances. By employing this principle we find:

$$
f(\mathbf{G}, \boldsymbol{\Omega})=\frac{n}{2 \pi(1+\lambda) \Theta_{t}} \frac{1}{\sqrt{2 \pi \Theta_{t}}}\left(\frac{I}{2 \pi m \Theta_{r}}\right)^{3 / 2} \exp \left[-\frac{1}{2} \frac{\left(\mathbf{G}_{t}-\mathbf{V}_{t}\right)^{2}}{(1+\lambda) \Theta_{t}}-\frac{G_{n}^{2}}{2 \Theta_{t}}-\frac{I \Omega^{2}}{2 m \Theta_{r}}\right]
$$

where $\mathbf{V}$ is the mean contact velocity, $\mathbf{V}=\langle\mathbf{G}\rangle$, which by definition only has components tangential to the wall. To emphasize this we will keep writing it as $\mathbf{V}_{t}$. Here and in the following, $\lambda$ is the granular temperature ratio, which for spheres is $\lambda=\frac{5 \Theta_{r}}{2 \Theta_{t}}$.

\section{Balances at the wall}

Jenkins ${ }^{16}$ derived the boundary conditions by considering an infinitesimal volume in the flow in the shape of a pillbox with one face fixed on the wall. We use the same balance of linear and angular momentum at the wall. As a result, two boundary tractions which are respectively the mean linear and angular collisional rate of the change of the particle momentum per unit area of the wall, are related to the shear stress in the flow by

$$
\begin{aligned}
& -\boldsymbol{\tau} \cdot \mathbf{n}=\mathbf{M} \\
& -\mathbf{m} \cdot \mathbf{n}=\mathbf{L}
\end{aligned}
$$


where $\boldsymbol{\tau}$ and $\mathbf{m}$ are the stress tensor and the couple stress in the flow. The quantities $\mathbf{M}$ and $\mathbf{L}$ are, respectively, the rates of supply of linear and angular momentum per unit area of the wall, and can be obtained as collisional integrals by $\mathbf{M}=\operatorname{Coll}(m \mathbf{c})$ and $\mathbf{L}=\operatorname{Coll}(\boldsymbol{I} \boldsymbol{\omega})$.

If $Q_{t}\left(=\mathbf{q}_{t} \cdot \mathbf{n}\right)$ and $Q_{r}\left(=\mathbf{q}_{r} \cdot \mathbf{n}\right)$ are the fluxes of translational and rotational fluctuation energy supplied by the wall to the flow, the balance of total energy for the pillbox is

$$
\mathbf{u} \cdot \boldsymbol{\tau} \cdot \mathbf{n}+\overline{\mathbf{\omega}} \cdot \mathbf{m} \cdot \mathbf{n}-\left(Q_{t}+Q_{r}\right)-D_{t}-D_{r}=0
$$

where $D_{t}$ and $D_{r}$ are the rate of dissipation of translational and rotational energy, due to the inelasticity of particle-wall collisions, per unit wall area. These can be obtained as collisional integrals by $-D_{t} \equiv \operatorname{Coll}\left(\frac{1}{2} m c^{2}\right),-D_{r} \equiv \operatorname{Coll}\left(\frac{1}{2} I \omega^{2}\right)$.

Combined with the above Eqs. 16a, b, we get

$$
\begin{aligned}
& Q_{t}=-\mathbf{u} \cdot \mathbf{M}-D_{t} \\
& Q_{r}=-\overline{\boldsymbol{\omega}} \cdot \mathbf{L}-D_{r}
\end{aligned}
$$

Here, the term on left hand side represents the flux of pseudothermal energy to the wall. The first term on the right hand side represents generation of pseudothermal energy by particle slip, and the last term is the rate of dissipation of this energy per unit area, due to the inelasticity of particle-wall collisions. Clearly, the wall can serve either as a source or a sink of pseudothermal energy, depending on the relative sizes of the terms on the right hand side.

\section{Collisional rate of change}

\section{Calculation of boundary tractions}

The normal stress supplied per unit area of the wall can be calculated by substituting $\psi=m c_{n}$ into Eq. 11, which has now been transformed to have integration variables $\mathbf{G}$ and $\mathbf{\Omega}$ :

$$
N=\operatorname{Coll}\left(m c_{n}\right)=-g_{0} \iint_{\mathbf{G} \cdot \mathbf{n} \leq 0} m \mathbf{J} \cdot \mathbf{n} f(\mathbf{G}, \mathbf{\Omega}) \mathbf{G} \cdot \mathbf{n} \mathrm{d} \mathbf{G} \mathrm{d} \mathbf{\Omega}
$$


After substituting Eqs. 7 and 15 into Eq. 19, and upon carrying out the above integration, the normal stress is found to be

$$
N=\frac{1}{2}(1+e) g_{0} m n \Theta_{t}
$$

This result comes as no surprise: it is exactly the pressure exerted by the wall onto the solid phase.

The component $S_{j}$ of the tangential stress can be obtained by applying $\psi=m c_{j}$ into Eq. 11,

$$
S_{j}=-\operatorname{Coll}\left(m c_{j}\right)=g_{0} \iint_{\mathbf{G} \cdot \mathbf{n} \leq 0} m \mathbf{J} \cdot \mathbf{j} f(\mathbf{G}, \mathbf{\Omega}) \mathbf{G} \cdot \mathbf{n} \mathrm{d} \mathbf{G} \mathrm{d} \mathbf{\Omega}
$$

The above integration is divided into two parts, which are contributions of sliding and sticking collisions. Using Eqs. 7 and 15, and integrating over all possibilities of $\boldsymbol{\Omega}$ and $G_{n}$ we find

$$
S_{j}=-\frac{(1+e) \mu g_{0} m n}{4 \pi(1+\lambda)} \int_{-\infty}^{\infty} \int_{-\infty}^{\infty} \frac{G_{j}}{G_{t}} \operatorname{erf}\left(\frac{G_{t} \cot \theta_{c}}{\sqrt{2 \Theta_{t}}}\right) \exp \left[-\frac{\left(G_{i}-V_{i}\right)^{2}+\left(G_{j}-V_{j}\right)^{2}}{2 \Theta_{t}(1+\lambda)}\right] d G_{i} d G_{j}
$$

where $i$ is the other tangential component, perpendicular to $j$. Note that $G_{t}=\sqrt{G_{i}^{2}+G_{j}^{2}}$ and $V_{t}=\sqrt{V_{i}^{2}+V_{j}^{2}}$. To perform the final integral, we first expand the error function into a Taylor series and then carry out the integration depending on the ratio $\sqrt{\frac{V_{t}^{2}}{2 \Theta_{t}(1+\lambda)}}$ as in Zhao et al. ${ }^{21}$. For details we refer to the Appendix. Each tangential stress component $S_{j}$ is found to scale with the contact velocity component $V_{j}$. Therefore the tangential stress will be oriented exactly opposite to the contact velocity. The ratio of total tangential stress $S$ to normal stress $N$ then reads:

$$
\frac{S}{N}=-\mu \cot \theta_{c} \frac{2 V_{t}}{\sqrt{2 \pi \Theta_{t}}} \max \left(A_{1}, A_{2}\right)
$$

where

$$
A_{1}=\frac{1}{2\left(1+X^{2}\right)}+\frac{Y^{2}\left(1-X^{2}\right)}{8 X^{2}\left(1+X^{2}\right)^{2}}+\frac{\left(4 X^{2}-Y^{2}\right) \arctan [X]}{8 X^{3}}
$$




$$
\begin{gathered}
A_{2}=\frac{\sqrt{\pi}}{2 Y} \operatorname{erf}(Y)-\frac{X^{2} \mathrm{e}^{-Y^{2}}\left(2 Y^{2}-1\right)}{4 Y^{2}}-\frac{\sqrt{\pi} X^{2}}{8 Y^{3}} \operatorname{erf}(Y) \\
X=\sqrt{1+\lambda} \cot \theta_{c} \\
Y=\sqrt{\frac{V_{t}^{2}}{2 \Theta_{t}}} \cot \theta_{c}
\end{gathered}
$$

For small particle slip velocity at the wall, the stress ratio is $\frac{S}{N}=-\mu \cot \theta_{c} \frac{2 V_{t}}{\sqrt{2 \pi \Theta_{t}}} A_{1}$, which corresponds to the "large friction/no sliding" limit by Jenkins ${ }^{16}$, while for large slip velocity the stress ratio is $\frac{S}{N}=-\mu \cot \theta_{c} \frac{2 V_{t}}{\sqrt{2 \pi \Theta_{t}}} A_{2}$, which corresponds to his "small friction/all sliding" limit. The max function in Eq. 23 ensures that the transition between these two regimes is continuous.

For rotational momentum change during collisions, the tangential stress can be calculated by substituting $\psi=I \omega_{j}$ into Eq. 11

$$
S_{\omega, j}=-\operatorname{Coll}\left(I \omega_{j}\right)=-\frac{\sigma}{2} m \mu g_{0}(1+e) \iint_{\mathbf{G} \cdot \mathbf{n} \leq 0}\left\{\begin{array}{c}
G_{n}^{2}(\mathbf{n} \times \mathbf{t}) \cdot \mathbf{j} \text {, sliding } \\
\mu_{0} G_{n} G_{t}(\mathbf{n} \times \mathbf{t}) \cdot \mathbf{j}, \text { sticking }
\end{array}\right\} f(\mathbf{c}, \boldsymbol{\omega}) d \mathbf{c} d \mathbf{\omega}
$$

Using the same integrating methods, we find

$$
S_{\omega, j}=\frac{(1+e)}{2} \sigma g_{0} \mu m n \Theta_{t} \cot \theta_{c} \frac{V_{n \times j}}{\sqrt{2 \pi \Theta_{t}}} \begin{cases}A_{1}, & \text { if } Y / X \text { is small } \\ A_{2}, \text { if } Y / X \text { is large }\end{cases}
$$

The normal angular stress is found to be 0 by substituting $\psi=I \omega_{n}$ into Eq. 11 . We can calculate the rotational heat flux changes in inserting Eq. 25 into Eq. 18b.

Figure 2 shows the results of our theory for the stress ratio versus normalized slip velocity $V_{t} / \sqrt{3 \Theta_{t}}$ for different collisional properties. To be able to make a comparison with literature results, in our theoretical expressions we set the granular temperature ratio $\lambda$ according to the theory of Jenkins and Zhang $^{26}$ (see Table 1). However note that in TFM simulations, $\lambda$ will be available directly by solving conservation equations for the translational and rotational granular temperatures. Figure 2 shows that 
generally we find better agreement with Louge's discrete particle simulation data ${ }^{5}$ in comparison with the theory from Schneiderbauer et al. ${ }^{20}$. Zhao et al. ${ }^{21}$ fitted logistic functions for $\lambda$ and obtained good agreement with Louge's data. They pointed out that the difference between using fitted and constant $\lambda$ is only obvious in the transition regime between the no sliding and all sliding limits. Figure 2(a) and 2(c) show that the present model can fit Louge's data very well for friction coefficient $\mu \leq 0.5$. As shown in Figure 2(c), for $\mu>0.5$ it is increasingly difficult to achieve large values of slip. Our theory underestimates the stress ratio in the regime where friction is very large and only small sliding occurs. In this regime, the assembly of spheres at the wall undergoes a phase transition from a dense gas to an ordered solid. Beyond this transition, the kinetic theory is not expected to be applicable ${ }^{5}$.

As shown in Figure 2(b), the present theory predicts the dependence of $\frac{1+e}{1+\beta} \frac{7 S}{2 N}$ on $\beta$ quite well for not too inelastic particles ( $e=0.9$ ). However, Figure 2(d) shows that for highly inelastic particles ( $\mathrm{e}=0.7$ and 0.5 ) our theory overestimates the stress ratio in the transition regime from sticking to sliding collisions. This may be due to the effect of a wall on the spatial and velocity distribution of particles in its vicinity. With decreasing normal restitution coefficient, anisotropy in the distribution of particle velocity fluctuations strongly increases, and particles experience significant structural changes. In this case the current kinetic theory, with central assumption of an isotropic granular temperature and homogeneous particle distribution, cannot correctly predict the absolute magnitude of normal stress 5 .

In summary, we find that the present theory is suitable for not too inelastic particle-wall collisions ( $e \geq 0.9$ ) which can vary from smooth to quite frictional ( $\mu \leq 0.5$ ).

\section{Calculation of rate of energy dissipation}

The rate of dissipation of translational energy, $D_{t}$, can be calculated by substituting $\psi=m c^{2} / 2$ into Eq. 11, which upon transformation of variables and combination with Eq. 8 gives 


$$
\begin{aligned}
-D_{t}= & -g_{0} \frac{n m}{2}\left(\frac{1}{2 \pi \Theta_{t}}\right)^{3 / 2}\left(\frac{I}{2 \pi m \Theta_{r}}\right)^{3 / 2} \iint_{\mathbf{G} \cdot \mathbf{n} \leq 0} \exp \left[-\frac{1}{2}\left(\frac{C^{2}}{\Theta_{t}}+\frac{I \Omega^{2}}{m \Theta_{r}}\right)\right] \\
& \left\{\begin{array}{l}
-\left(1-e^{2}\right) G_{n}{ }^{2}+\mu(1+e)\left\{\begin{array}{c}
2 G_{n} \mathbf{c} \cdot \mathbf{t}+\mu(1+e) G_{n}{ }^{2}, \text { sliding } \\
2 \mu_{0} G_{t} \mathbf{c} \cdot \mathbf{t}+\mu(1+e) \mu_{0}{ }^{2} G_{t}{ }^{2}, \text { sticking }
\end{array}\right\} \mathbf{G} \cdot \mathbf{n d} \mathbf{G} \mathrm{d} \mathbf{\Omega}
\end{array}\right.
\end{aligned}
$$

The above equation calculates the contributions of sliding and sticking collisions separately. Again we first perform the integrals over $\boldsymbol{\Omega}$ and $G_{n}$. Despite several efforts, the remaining integrals could not be evaluated exactly, so they are carried out to order $O\left(\frac{Y^{2}}{X^{2}}\right)$ (details can be found in the Appendix).

The resulting approximated energy dissipation rate is

$$
\begin{aligned}
-D_{t}= & g_{0} n m \Theta_{t} \sqrt{\frac{\Theta_{t}}{2 \pi}}\left\{-\left(1-e^{2}\right)+\mu^{2}(1+e)^{2}\left(1-\frac{e^{-\frac{Y^{2}}{1+X^{2}}}}{1+X^{2}}\right)\right\} \\
& +g_{0} \mu \mathrm{nm}(1+e) \Theta_{t} \cot \theta_{c}\left\{\begin{array}{l}
(1+\lambda) \sqrt{\frac{2 \Theta_{t}}{\pi}} B_{1}+\frac{\sigma}{2} \frac{(\mathbf{V} \times \overline{\boldsymbol{\omega}}) \cdot \mathbf{n}}{\sqrt{2 \pi \Theta_{t}}} A_{1}, \text { if } Y / X \text { is small } \\
\sqrt{\frac{2 \Theta_{t}}{\pi}} \frac{V_{t}^{2}}{2 \Theta_{t}} B_{2}+\frac{\sigma}{2} \frac{(\mathbf{V} \times \overline{\boldsymbol{\omega}}) \cdot \mathbf{n}}{\sqrt{2 \pi \Theta_{t}}} A_{2}, \text { if } Y / X \text { is large }
\end{array}\right.
\end{aligned}
$$

Here $A_{1}$ and $A_{2}$ have been calculated before, and $B_{1}$ and $B_{2}$ are given by

$$
\begin{aligned}
& B_{1}=\frac{2 X^{2}\left(X^{2}+1\right)+Y^{2}\left(X^{2}-Y^{2}+3\right)}{4 X^{2}\left(1+X^{2}\right)^{2}}+\frac{3 Y^{4}\left(1+7 X^{4}\right)}{96 X^{4}\left(1+X^{2}\right)^{3}}+\frac{8 X^{2}\left(2 X^{2}+Y^{2}\right)-Y^{4}}{32 X^{5}} \arctan X \\
& B_{2}=\frac{\sqrt{\pi}}{2 Y} \operatorname{erf}(Y)+\frac{X^{2} \sqrt{\pi}}{8 Y^{3}} \operatorname{erf}(Y)+\frac{3-2 Y^{2}}{4 Y^{2}} X^{2} \mathrm{e}^{-Y^{2}}+\frac{\sqrt{\pi} X^{4}}{64 Y^{5}} \operatorname{erf}(Y)
\end{aligned}
$$

Note that while performing the integrals, we did not neglect the projected particle spin $\boldsymbol{\omega} \cdot(\mathbf{n} \times \mathbf{t})$. As a result, the calculated energy dissipation rate is not only a function of the contact point slip velocity but also of the average angular velocity $\overline{\boldsymbol{\omega}}$. In the expression for $D_{t}$, this results in the term proportional to $(\mathbf{V} \times \overline{\boldsymbol{\omega}}) \cdot \mathbf{n}$. In our comparisons with DPM we will use the correlation $\overline{\boldsymbol{\omega}} \approx-(\mu / \sigma) \mathbf{u} \times \mathbf{n}$. 
The rate of dissipation of rotational energy, $D_{r}$, can be calculated by substituting $\psi=I \omega^{2} / 2$ into Eq. 11, which upon transformation of variables and combining with Eq. 9 gives

$$
\begin{aligned}
& -D_{r}=-\frac{g_{0} \sigma m}{2} \mu(1+e) n\left(\frac{1}{2 \pi \Theta_{t}}\right)^{3 / 2}\left(\frac{I}{2 \pi m \Theta_{r}}\right)^{3 / 2} \\
& \iint_{\mathbf{G} \cdot \mathbf{n} \leq 0}\left\{\begin{array}{c}
-\boldsymbol{\omega} \cdot(\mathbf{n} \times \mathbf{t}) G_{n}+\frac{m \sigma}{4 I} \mu(1+e) G_{n}{ }^{2}, \text { sliding } \\
-\boldsymbol{\omega} \cdot(\mathbf{n} \times \mathbf{t}) \mu_{0} G_{t}+\frac{m \sigma}{4 I} \mu \mu_{0}{ }^{2}(1+e) G_{t}{ }^{2}, \text { sticking }
\end{array} \exp \left[-\frac{1}{2}\left(\frac{C^{2}}{\Theta_{t}}+\frac{I \Omega^{2}}{m \Theta_{r}}\right)\right] \mathbf{G} \cdot \mathbf{n d} \mathbf{G d} \mathbf{\Omega}\right.
\end{aligned}
$$

Again we have to make similar approximations. As shown in the appendix, the approximated rotational energy dissipation which considers both sliding and sticking contributions is

$$
\begin{aligned}
-D_{r}= & \frac{5 g_{0} n m}{2} \mu^{2}(1+e)^{2} \Theta_{t} \sqrt{\frac{\Theta_{t}}{2 \pi}}\left(1-\frac{e^{-\frac{Y^{2}}{1+X^{2}}}}{1+X^{2}}\right) \\
& -\frac{\sigma}{2} g_{0} n m \mu(1+e) \Theta_{t} \cot \theta_{c} \frac{(\mathbf{V} \times \overline{\boldsymbol{\omega}}) \cdot \mathbf{n}}{\sqrt{2 \pi \Theta_{t}}}\left\{\begin{array}{l}
A_{1}, \text { if } Y / X \text { is small } \\
A_{2}, \text { if } Y / X \text { is large }
\end{array}\right.
\end{aligned}
$$

The total rate of energy dissipation can be obtained by adding Eqs. 27 and 29. Jenkins and Louge ${ }^{17}$ and Zhao et al. ${ }^{21}$ made an additional correction to account for the effect of particle-particle collisions. Including their correction, we find for the total rate of energy dissipation:

$$
\begin{aligned}
-D= & g_{0} n m \Theta_{t} \sqrt{\frac{\Theta_{t}}{2 \pi}}\left\{-\left(1-e^{2}\right)+\frac{7}{2} \mu^{2}(1+e)^{2} \frac{2-e e_{p}}{2+e e_{p}}\left(1-\frac{e^{-\frac{Y^{2}}{1+X^{2}}}}{1+X^{2}}\right)\right\} \\
& +(1+e) \mu g_{0} n m \Theta_{t} \cot \theta_{c}(1+\lambda) \sqrt{\frac{2 \Theta_{t}}{\pi}}\left[\frac{B_{1}, \text { if } Y / X \text { is small }}{2 \Theta_{t}(1+\lambda)} B_{2} \text {, if } Y / X \text { is large }\right]
\end{aligned}
$$

where $e_{p}$ is the coefficient of normal restitution for particle-particle collisions (remember that $e$ is for particle-wall collisions). 
Figure 3 shows the normalized dissipation $D / \sqrt{3 \Theta_{t} N^{2}}$, which can be evaluated from Eqs. 20 and 30 . As before, for frictions $\mu \leq 0.5$ our theory agrees very well with the discrete particle simulation data of Louge ${ }^{5}$ and shows improvement over the theory of Schneiderbauer et al. ${ }^{20}$, especially in the transition regime from sticking to sliding. For very high friction $\mu>0.5$ our theory underestimates the amount of energy dissipation.

\section{Fluctuation heat flux}

The total heat flux (flux of total fluctuation energy) from the wall to the granular flow can be obtained from Eqs. 18a, b and 30,

$$
\frac{Q}{N \sqrt{3 \Theta_{t}}}=\sqrt{\frac{2}{3 \pi}}\left\{\begin{array}{l}
\mu \cot \theta_{c}(1+\lambda) \min \left[2\left(B_{1}-\frac{V_{t}^{2}}{2 \Theta_{t}(1+\lambda)} A_{1}\right), \frac{V_{t}^{2}}{\Theta_{t}(1+\lambda)}\left(B_{2}-A_{2}\right)\right] \\
+\frac{7}{2} \mu^{2}(1+e) \frac{2-e e_{p}}{2+e e_{p}}\left(1-\frac{e^{-\frac{Y^{2}}{1+X^{2}}}}{1+X^{2}}\right)-(1-e)
\end{array}\right\}
$$

Figure 4 shows the approximated normalized heat flux versus normalized slip velocity for different collisional properties. The flux can be negative or positive depending on the slip velocity. This may be analyzed from Eq. 18(a, b). The heat flux contains two competing terms: a source term due to the transfer of kinetic energy from the average particle flow to velocity fluctuations induced by wall roughness, and a sink term representing the dissipation by inelastic particle-wall collisions. For relatively small slip velocities $\left(V_{t} / \sqrt{3 \Theta_{t}}<10\right)$, the dissipation due to inelastic particle-wall collisions is dominant; otherwise, the wall roughness is dominant. As expected, for not too inelastic particles ( $e \geq 0.9$ ), our theory obtains better agreement with Louge's data than the theory from Schneiderbauer et al. ${ }^{20}$, whereas both theories are inaccurate for $e<0.9$. Figure 4(a) shows that with increasing friction coefficient $\mu$, the heat flux becomes more negative in the 'small sliding' regime and more positive in the 'large sliding' regime. Figure 4(b) shows that according to our theory the influence of tangential restitution coefficient $\beta$ on the heat flux is not very pronounced, similar to the discrete particle 
simulation data of Louge ${ }^{5}$. Our predictions are closer to this simulation data than the predictions from the theory of Schneiderbauer et al. ${ }^{20}$, but still deviate somewhat at intermediate slip velocities. Figure 4(c) shows that large deviations occur in both theories when decreasing the normal restitution coefficient below a value of 0.9 .

We can also determine the separate fluxes of translational and rotational fluctuation energy:

$$
\begin{aligned}
& \frac{Q_{t}}{N \sqrt{3 \Theta_{t}}}=(1+\lambda) \mu \cot \theta_{c} \sqrt{\frac{8}{3 \pi}} \min \left[\left(B_{1}-\frac{V_{t}^{2} A_{1}}{2 \Theta_{t}(1+\lambda)}\right), \frac{V_{t}^{2}\left(B_{2}-A_{2}\right)}{2 \Theta_{t}(1+\lambda)}\right] \\
&+\sqrt{\frac{2}{3 \pi}}\left\{\mu^{2}(1+e) \frac{2-e e_{p}}{2+e e_{p}}\left(1-\frac{e^{-\frac{Y^{2}}{1+X^{2}}}}{1+X^{2}}\right)-1+e\right\} \\
& Q_{r}=\frac{5}{4}(1+e)^{2} \mu^{2} m n g_{0} \Theta_{t} \frac{2-e e_{p}}{2+e e_{p}} \sqrt{\frac{2 \Theta_{t}}{\pi}}\left(1-\frac{e^{-\frac{Y^{2}}{1+X^{2}}}}{1+X^{2}}\right)
\end{aligned}
$$

Figure 5 shows that normalized translation and rotational fluxes versus the normalized slip velocity. This is the first time, to the best of our knowledge, that these separate fluxes have been shown. For the normalized translational heat flux, both negative and positive values can be obtained, depending on the competition between wall roughness and the dissipation by inelastic particle-wall collisions. We find that the influence of friction coefficient on the translational fluctuation energy is similar to that of the total fluctuation energy. The rotational heat flux is always positive which means that the wall serves as a source of rotational fluctuation energy. Besides, the non-zero assumption of mean angular velocity at the wall also contributes to the positive rotational heat flux. Furthermore, the rotational fluctuation energy increases with increasing friction coefficient, which implies that particles tend to spin more adjacent to the wall with increasing friction.

\section{Numerical implementation of wall boundary conditions}


The new boundary conditions have been implemented in our in-house two-fluid model code, in which a staggered grid is employed. Here we give a detailed description of the numerical implementation of the boundary conditions for the solid phase slip velocity and the fluctuation energy.

For computing the solid wall shear stress and fluctuation energy wall fluxes, we use a first order gradient approximation between the computed variables and the fictitious imposed variables at the wall (see Figure 6).

According to the tangential shear stress in $i$ direction, the flat frictional wall boundary conditions for the solids phase velocity can be written as $-\mathbf{i} \cdot(\boldsymbol{\tau} \cdot \mathbf{n})=-\mathbf{i} \cdot \mathbf{S}$, where $\boldsymbol{\tau}$ is the solids stress tensor. It can be easily found that the stress boundary condition then translates to the following requirement on the solid velocity gradient:

$$
\left(\mu_{t_{s}}+\mu_{r_{s}}\right) \frac{\partial v_{i}}{\partial x_{n}}=g_{0} \mu m n(1+e) \cot \theta_{c} \frac{\Theta_{t} V_{i}}{\sqrt{2 \pi \Theta_{t}}} \max \left(A_{1}, A_{2}\right)
$$

Here $\mu_{t_{s}}, \mu_{r_{s}}$ are the translational and rotational shear viscosities respectively ${ }^{27}$. On the right-hand side, the components of the local mean contact velocity $V_{i}$ are expressed in terms of interpolated values of the solids velocities, where the correlation for the average angular velocity which was obtained from DPM simulations is used. The gradient at the left-hand side is also expressed as an interpolated value. For example, in Figure 6, the $y$-velocity components in the neighborhood of the right wall can be written as follows

$$
\left(v_{s, y}\right)_{0, j+\frac{1}{2}, k}=\mathrm{C}_{1}\left(v_{s, y}\right)_{1, j+\frac{1}{2}, k}+\mathrm{D}_{1}\left(v_{s, y}\right)_{2, j+\frac{1}{2}, k}
$$

Where $\mathrm{C}_{1}, \mathrm{D}_{1}$ are pre-factors depending on the interpolation method and calculated based on Eq. 33 . $\left(v_{s, y}\right)_{1, j+\frac{1}{2}, k}$ and $\left(v_{s, y}\right)_{2, j+\frac{1}{2}, k}$ are solids velocities in computational cells 1 and 2 .

Since we have calculated the translational fluctuation energy at the wall, the corresponding boundary conditions can be obtained from the balance of the fluctuation energy

$$
\kappa_{t} \frac{\partial \Theta_{t}}{\partial n}=Q_{t}
$$


where $\kappa_{t}$ is the particle pseudo thermal conductivity ${ }^{27}$ and $Q_{t}$ is the pseudo heat flux in this work. In the direction normal to the right wall, the final boundary condition for translational granular temperature in numerical code is

$$
\left(\Theta_{t}\right)_{0, j+\frac{1}{2}, k}=\mathrm{C}_{2}\left(\Theta_{t}\right)_{1, j+\frac{1}{2}, k}+\mathrm{D}_{2}\left(\Theta_{t}\right)_{2, j+\frac{1}{2}, k}+\mathrm{E}_{2}
$$

where $C_{2}, D_{2}, E_{2}$ are pre-factors depending on the interpolation method.

Similarly, the boundary condition for rotational granular temperature can be obtained by

$$
\kappa_{r} \frac{\partial \Theta_{t}}{\partial n}=Q_{r}
$$

where $\kappa_{r}$ is the particle pseudo thermal conductivity ${ }^{27}$ and $Q_{r}$ is the rotational pseudo heat flux in this work. The boundary condition in numerical code is written as

$$
\left(\Theta_{r}\right)_{0, j+\frac{1}{2}, k}=\mathrm{C}_{3}\left(\Theta_{r}\right)_{1, j+\frac{1}{2}, k}+\mathrm{D}_{3}\left(\Theta_{r}\right)_{2, j+\frac{1}{2}, k}+\mathrm{E}_{3}
$$

Where $\mathrm{C}_{3}, \mathrm{D}_{3}, \mathrm{E}_{3}$ are again pre-factors depending on the interpolation method.

Note that the above BCs for solid velocity (Eq. 33) and granular temperature (Eq. 35) can not only be used in the KTGF of Yang et al. ${ }^{27}$, but also can be applied in other kinetic theories with only change regarding solid shear viscosity and thermal conductivity.

\section{Results and discussion}

We will now test the performance of our new boundary conditions. For the solids bulk rheology we will use the kinetic theory of rough spheres described by Yang et al. ${ }^{27}$, using the same settings as used in the validation case by Yang et al. ${ }^{32}$. However in the latter work, in the absence of the boundary conditions theory described in this paper, the often-used BCs of Sinclair and Jackson ${ }^{12}$ (with a specularity coefficient $\varphi=0.1$ ) were applied, and adiabatic conditions on the rotational heat flux were assumed. In the following, the simulation results using our new boundary conditions are compared with 
these previous TFM results, and additionally with results from more detailed DPM simulations, and experimental data obtained from combined PIV-DIA measurements on a quasi-2D bubbling fluidized bed containing $3 \mathrm{~mm}$ glass particles ${ }^{33}$. Details about this system can be found in Table 2 .

First, time-averaged solids fraction distributions from the three models and PIV-DIA experiments are shown in Figure 7. Compared with the old BCs, Figure 7(c), for the new BCs, Figure 7(d), the dense solids zone moves more along the wall. This larger spreading of the high density zone along the walls can also be found in DPM simulations, Figure 7(b), and in experiments, Figure 7(a). We can therefore conclude that the new BCs improve the two-fluid model predictions in terms of the solids fraction distribution.

Figure 8 shows the vertical and horizontal profiles of particle concentration in the bed for the different models. The width-averaged height profile in Figure 8(a) shows that at the bottom (height $<0.2$ ), the simulated particle concentration from TFM is lower than that obtained from the DPM simulation, while at height evaluation (0.2-0.4 m), the simulated particle concentration obtained from TFM is higher. In this width-averaged fashion, the difference between the TFM simulations with different BCs is small. Figure 8(b) shows that both TFM simulations underestimate the solids volume fraction in the center and overestimate the solids concentration in the near-wall region.

Figure 9 shows the time-averaged solids circulation patterns obtained from PIV-DIA experiments, DPM and TFM simulations. In Figures 9(c) and 9(d) (the TFM simulations) the solids axial velocity close to the bottom walls is overestimated compared to that of Figure 9(b) (DPM simulation). This explains why in Figures 7 and 8 the TFM simulations were found to predict larger zones of intermediate solids volume fraction 0.42-0.48 near the bottom and the side walls in comparison with the more detailed DPM simulations. However, when comparing the TFM simulation results using Sinclair and Jackson BCs with TFM using the new BCs, we clearly see that the new BCs lead to better agreement with the DPM simulation, not only in the central part of the bed but also at the bottom zone. The TFM employing new BCs, Figure 9(d), produces a more pronounced circulation pattern, which is in closer agreement with that from DPM simulations, Figure 9(b), as a result of the increase in energy dissipation prevailing during particle-wall collisions. We note that the predictions of the TFM with the new BCs 
are still not in perfect agreement with the DPM simulations. This may be attributed to the failure of our model to accurately predict the particle stress and low velocity fluctuations occurring in some very dense zones near the walls where long enduring multi-particle contacts occur. In our future work we will also compare our method for much faster fluid-particle flows, in which case we expect a better agreement.

A comparison of the distribution of translational granular temperature predicted by the three models is shown in Figure 10. The TFM simulation with the new BCs predicts a reduction in the zones of high granular temperature values compared to TFM simulation results employing Sinclair and Jackson BCs. In the dense region at the bottom, high collision rates cause strong dissipation of fluctuating energy, and all models reach the minimum granular temperature limit. The zones of low granular temperature in Figure 10(c) agree better with the DPM predictions in Figure 10(a). In TFM simulation using the new BCs, the wall serves as a sink term for the particle fluctuation energy, i.e. energy is predominantly dissipated during particle-wall collisions. Note that both TFM models predict high granular temperatures in the splash zone above the bed, which is not observed in the discrete particle simulation. On the one hand, the number of particles on which the granular temperature evaluation is based is too small to obtain a statistically meaningful average in this region for the discrete particle model ${ }^{34}$. On the other hand, in the very dilute regime, the solid phase velocity fluctuations are no longer dominated by kinetic and collisional influences, but by the interaction with the gas phase. The gas phase turbulence may play a significant role in this region, thus neglecting turbulence in this region is questionable in general $^{35}$. We will therefore compare granular temperatures (in different areas) below the splash zone in the next part.

Figure 11 shows contour plots of the rotational granular temperatures obtained from both TFM models and DPM. The rotational granular temperature in the old TFM model, Figure 11(b), shows a very homogeneous distribution. This is a consequence of imposing adiabatic boundary conditions on the flux of rotational fluctuation energy in combination with the assumption of zero average angular velocity. Using the new BCs, the rotational granular temperature distribution becomes more inhomogeneous, with higher rotational granular temperatures in the center of the bed and lower 
rotational granular temperatures near the walls and bottom of the bed, in better qualitative agreement with DPM simulations. There is no quantitative agreement yet, probably because of the assumption of zero average angular velocity in the bulk of the bed ${ }^{27}$. Nevertheless, note that the overall magnitude of the rotational granular temperature agrees to a reasonable extent (we will analyse the magnitude of the rotational granular temperature in detail in the next part).

Figures 12 and 13 show the granular temperature averaged over different areas as a function of time. Figure 12(a) shows that the value of the granular temperature at the bottom is lower than in other parts of the fluidized bed. There exists a deviation between TFM models on the one side and the DPM model on the other side. This deviation has several possible reasons. First, we use large particles (diameter 3 $\mathrm{mm})$. For the energy equation in the dense region, a correction for the damping term is needed, similar to the use of a frictional stress for dense packings. Furthermore, in the limit of maximum packing the granular flow becomes incompressible and the solids pressure decouples from the solids volume fraction. In frictional flow, particles are in continuous contact and momentum transfer occurs mainly through enduring frictional contacts. Dense packing and particle roughness result in changes in the collision frequency and velocity distribution function (Chialvo and Sundaresan introduced a "chain length” to the expression for the energy dissipation rate ${ }^{36}$ ). In this case, kinetic theory of granular breaks down.

With increasing height, the values of the granular temperature also increase, see Figure 12(b) and 12(c), and the overall agreement between TFM models and DPM becomes better (area 0.2-0.3 and 0.30.4). It is apparent that the TFM with the new BCs shows better agreement with DPM simulations in these areas. In Figure 12(d) we study the average rotational granular temperature as a function of time. The TFM models are found to overpredict the rotational granular temperature by a factor of 2 .

Next we focus on different vertical areas, dividing between near-wall and bulk areas. Figure 13(a) shows that in the near-wall region the agreement with DPM is almost quantitative, but with larger positive fluctuations as a function of time. Figure 13(b) shows that in the bulk region the fluctuations are similar to those observed in DPM, but the average (as observed above) is overpredicted by a factor of 2. 
A quantitive comparison of the granular temperatures is shown in Table 3. From the table, and from the observations above, we can conclude that the new BCs lead to an improvement in the agreement with detailed DPM simulations.

Figure 14 shows a comparison of the granular temperature profiles from different models versus solids concentration. The granular temperature in all cases reaches a maximum around solids concentration $0.3( \pm 0.025)$. Then, the predicted granular temperature from all models decreases with increasing solids concentration as a result of the rapid increase of the rate of dissipative particle collisions in the dense regime. In the region with solids concentration from 0.25-0.35 and dense region (solids concentration $>0.5$ ), the simulated granular temperature from the TFM with new BCs is lower than that of the TFM with Sinclair and Jackson BCs, while for solids concentration from 0.4-0.5 both TFM models predict similar granular temperatures. As can be seen from the figure, overall the new BCs lead to better agreement with DPM simulations. This verifies that appropriate BCs can indeed improve our simulation results in the dilute and very dense regimes, as pointed out by Yang et al. ${ }^{32}$.

Figure 15 shows a comparison of time-averaged axial particle velocity components at different heights. The axial particle velocity is positive in the center and negative near the walls, which indicates that particles move upward in the center and flow down near the wall. There are small deviations near the wall for both TFM models compared with DPM simulations. At the center, the TFM model with Sinclair and Jackson boundary conditions predicts a higher velocity magnitude than the TFM model with the new BCs. The TFM simulation results with the new BCs are in better agreement with DPM simulations.

Figure 16 shows a comparison of the solids axial mass flux. There is a lower downwards mass flux predicted by the TFM simulations in the near-wall region. This is due to the deviation between TFM and DPM simulations for the prediction of solids volume fraction near the wall shown in Figure 8(b). However, we can see that TFM with present BCs is better at predicting the particle axial velocity in some cases. Thus, the BCs based on the particle-wall collision mechanism can be used to predict wall effects. 


\section{Conclusions}

We have derived a theory for boundary conditions (BCs) on momentum and energy transfer between a granular flow and a flat frictional wall. The theory distinguishes between sliding and sticking collisions and includes particle rotation. New expressions for the solids stress tensors, energy dissipation rates per unit area and the fluxes of fluctuation energy are proposed. The new BCs are validated by comparing with literature data from Louge ${ }^{5}$ for stress ratio and heat fluxes using a constant value for the ratio of rotational to translational granular temperatures from Jenkins and Zhang ${ }^{26}$. The results show that our theory works excellent for not too inelastic particles with $e \geq 0.9$ and smooth to quite frictional walls ( $\mu \leq 0.5$ ). We also implemented the new BCs into our in-house two fluid model code incorporating bulk particle rheology based on kinetic theory of granular flows of rough spheres by Yang et al. ${ }^{27}$. Simulations of a pseudo-2D fluidized bed using this new two fluid model show that the new BCs are often better capable of predicting solids axial velocity profiles, solids distribution near the walls and granular temperatures than two fluid model simulations based on Johnson and Sinclair BCs. In very dense zones long enduring particle contacts occur, leading to multi-particle collisions. We have observed that, generally, in these zones all TFM models (irrespective of BCs) make the largest relative error in overpredicting the granular temperature. We expect that this will be less of an issue in particle flows occurring at elevated fluidization velocities because there will be less very dense zones. We will investigate this in our future work.

\section{Acknowledgement}

The authors thank the European Research Council for its financial support, under its Advanced Investigator Grant scheme, contract no. 247298 (Multi-scale Flows).

\section{Notation}

c translational particle velocity, $\mathrm{m} / \mathrm{s}$

$\mathbf{v}$ mean particle translational velocity, $\mathrm{m} / \mathrm{s}$ 


\begin{tabular}{|c|c|}
\hline $\mathbf{C}$ & translational fluctuating particle velocity, m/s \\
\hline c' & translational particle velocity after collision, m/s \\
\hline $\mathbf{G}$ & relative particle velocity at contact point, $\mathrm{m} / \mathrm{s}$ \\
\hline $\mathbf{J}$ & impulse force, $\mathrm{kg} \mathrm{m} / \mathrm{s}$ \\
\hline $\mathbf{k}$ & unit normal vector at contact point \\
\hline j & unit tangential vector at contact point \\
\hline g & gravitational acceleration, $\mathrm{m} / \mathrm{s}^{2}$ \\
\hline $\mathbf{r}$ & position of the particle, $\mathrm{m}$ \\
\hline$f$ & single particle distribution function \\
\hline$I$ & moment of inertia, $\mathrm{kg} \mathrm{m}^{2}$ \\
\hline$m$ & mass of the particle, $\mathrm{kg}$ \\
\hline$n$ & particle number density, $\mathrm{m}^{-3}$ \\
\hline$U_{m f}$ & minimum fluidization velocity, $\mathrm{m} / \mathrm{s}$ \\
\hline$e$ & normal restitution coefficient \\
\hline$Q$ & heat flux, $\mathrm{kg} / \mathrm{s}^{3}$ \\
\hline$D$ & energy dissipation rate, $\mathrm{kg} / \mathrm{s}^{3}$ \\
\hline$g_{0}$ & radial distribution function \\
\hline $\mathbf{M}$ & linear momentum per unit area of the wall, $\mathrm{kg} /\left(\mathrm{ms}^{2}\right)$ \\
\hline $\mathbf{L}$ & angular momentum per unit area of the wall, $\mathrm{kg} /\left(\mathrm{ms}^{2}\right)$ \\
\hline
\end{tabular}

\section{Greek symbols}

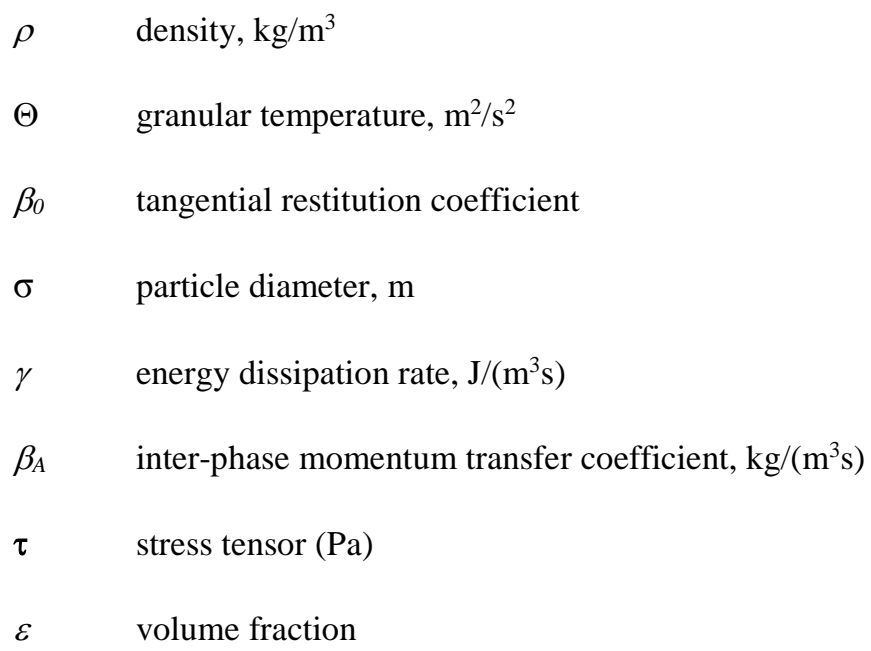


$\kappa \quad$ thermal conductivity, $\mathrm{kg} /(\mathrm{m} \cdot \mathrm{s})$

$\chi_{c} \quad$ collisional source of particle properties

$\theta_{c} \quad$ collisional flux of particle properties

$\mathbf{\Omega} \quad$ rotational fluctuating particle velocity, $\mathrm{rad} / \mathrm{s}$

$\mu_{t} \quad$ translational shear viscosity, $\mathrm{kg} /(\mathrm{ms})$

\section{Subscripts}

1 particle 1

2 particle 2

S solid phase

$r \quad$ rotational contribution

$i \quad$ coordinate direction $i$

$j \quad$ coordinate direction $j$

$k \quad$ coordinate direction $k$

n normal direction

w wall

\section{Literature Cited}

1. Hanes DM, Inman DL. Observations of rapidly flowing granular-fluid materials. J Fluid Mech. 1985; 15: 357-380

2. Sommerfeld M, Huber N. Experimental analysis and modelling of particle-wall collisions. Int $J$ Multiphase Flow. 1999; 25(6): 1457-1489.

3. Benyahia S, Syamlal M, O'Brien TJ. Evaluation of boundary conditions used to model dilute, turbulent gas/solids flows in a pipe. Powder Technol. 2005; 156(2): 62-72.

4. Loha C, Chattopadhyay H, Chatterjee PK. Euler-Euler CFD modeling of fluidized bed: Influence of specularity coefficient on hydrodynamic behavior. Particuology. 2013; 11(6): 673-680. 
5. Louge MY. Computer-simulations of rapid granular flows of spheres interacting with a flat, frictional boundary. Phys Fluids. 1994; 6(7): 2253-2269.

6. Hui K, Ungar JE, Haff PK, Jackson R. Boundary conditions for high-shear grain flows. J Fluid Mech. 1984; 145: 223-233.

7. Jenkins J, Richman MW. Boundary conditions for plane flows of smooth, nearly elastic, circular disks. J Fluid Mech. 1986; 171: 53-69.

8. Johnson PC, Jackson R. Frictional-collisional constitutive relations for granular materials, with application to plane shearing. J Fluid Mech. 1987; 176: 67-93.

9. Almuttahar A, Taghipour F. Computational fluid dynamics of high density circulating fluidized bed riser: Study of modeling parameters. Powder Technol. 2008; 185(1): 11-23.

10. Zhou X, Gao J, Xu C, Lan X. Effect of wall boundary condition on CFD simulation of CFB risers. Particuology. 2013; 11(5): 556-565.

11. Li T, Grace J, Bi X. Study of wall boundary condition in numerical simulations of bubbling fluidized beds. Powder Technol. 2010; 203(3): 447-457.

12. Sinclair J, Jackson R. Gas-Particle Flow in a Vertical Pipe with Particle-Particle Interactions. AIChE J. 1989; 35 (9): 1473-1486.

13. Nieuwland JJ, van Sint Annaland M, Kuipers JAM, Van Swaaij WPM. Hydrodynamic modeling of gas/particle flows in riser reactors. AIChE J. 1996; 42(6): 1569-1582.

14. Lu HL, Gidaspow D. Hydrodynamics of binary fluidization in a riser: CFD simulation using two granular temperatures. Chem Eng Sci. 2003; 58(16): 3777-3792.

15. Verma V, Padding JT, Deen NG, Kuipers JAM. Bubble formation at a central orifice in a gas-solid fluidized bed predicted by three-dimensional two-fluid model simulations. Chem Eng J. 2014; 245: 217-227.

16. Jenkins JT. Boundary-conditions for rapid granular flow-flat, frictional walls. J Appl Mech. 1992; 59(1): 120-127.

17. Jenkins JT, Louge MY. On the flux of fluctuation energy in a collisional grain flow at a flat, frictional wall. Phys Fluids. 1997; 9(10): 2835-2840. 
18. Konan NA, Simonin O, Squires KD. Rough wall boundary condition derivation for particle continuum equations: validation from LES/DPS of gas-solid turbulent channel flow. In: Proceedings of the $7^{\text {th }}$ International Symposiumon Numerical Methods for Multiphase Flows. ASME Joint U.S. European Fluids Engineering Summer Meeting, FEDSM2006-98267. 2006; 1723-1732.

19. Goldhirsch I. Rapid granular flows. Annu Rev Fluid Mech. 2003; 35(1): 267-293.

20 Schneiderbauer S, Schellander D, Loderer A, Pirker S. Non-steady state boundary conditions for collisional granular flows at flat frictional moving walls. Int J Multiphase Flow. 2012; 43: 149-156.

21. Zhao Y, Zhong Y, He Y, Schlaberg H.I. Boundary conditions for collisional granular flows of frictional and rotational particles at flat walls. AIChE J. 2014; 60(12): 4065-4075.

22. Li TW, Benyahia S. Revisiting Johnson and Jackson boundary conditions for granular flows. AIChE J. 2012; 58(7): 2058-2068.

23. Soleimani A, Pirker S, Schneiderbauer S. Solid boundary condition for collisional gas-solid flows at rough walls. Powder Technol. 2015; 281: 28-33.

24. Fede P, Simonin O, Ingram A. 3D numerical simulation of a lab-scale pressurized dense fluidized bed focussing on the effect of the particle-particle restitution coefficient and particle-wall boundary conditions. Chem Eng Sci. 2016; 142: 215-235.

25. Sakiz M, Simonin O. Development and validation of continuum particle wall boundary conditions using Lagrangian simulation of a vertical gas-solid channel flow. In: Proceedings of the ASME Fluids Engineering Division Summer Meeting, FEDSM, 1999; 99-7898.

26. Jenkins J, Richman MW. Boundary conditions for plane flows of smooth, nearly elastic, circular disks. J Fluid Mech. 1986; 171: 53-69.

27. Yang L, Padding JT, Kuipers JAM. Modification of Kinetic Theory for Frictional Spheres, Part I: Two-fluid model derivation and numerical implementation. Chem Eng Sci. 2016; 152: 767-782.

28. McCoy BJ, Sandler SI, Dahler JS. Transport properties of polyatomic fluids. IV. The kinetic theory of a dense gas of perfectly rough spheres. J Chem Phys. 1966; 45(10): 3845-3512.

29. Ergun S. Fluid flow through packed columns. Chem Eng Prog. 1952; 48: 89-94.

30. Wen CY, Yu YH. A generalized method for predicting the minimum fluidization velocity. AIChE J. 1966; 12(3): 610-612. 
31. Srivastava A, Sundaresan S. Analysis of a frictional-kinetic model for gas-particle flow. Powder Technol. 2003; 129: 72-85.

32. Yang L, Padding JT, Kuipers JAM. Modification of Kinetic Theory of Granular Flow for Frictional Spheres, Part II: Model validation. Chem Eng Sci. 2016; 152: 783-794.

33. Buist KA, Deen NG, Kuipers JAM. Improved magnetic particle tracking technique in dense gas fluidized beds. AIChE J. 2014; 60(9): 3133-3142.

34. Goldschmidt MJV, Kuipers JAM, Van Swaaij WPM. Hydrodynamic modelling of dense gasfluidised beds using the kinetic theory of granular flow: effect of coefficient of restitution on bed dynamics. Chem Eng Sci. 2001; 56(2): 571-578.

35. Boemer A, Qi H, Renz U. Eulerian simulation of bubble formation at a jet in a two-dimensional fluidized bed. Int J Multiphase Flow. 1997; 23(5): 927-944.

36. Chialvo S, Sundaresan S. A modified kinetic theory for frictional granular flows in dense and diluteregimes. Phys Fluids. 2013; 25(7): 070603.

\section{Appendix}

Calculation of shear stress tensor

Based on the expression for the shear stress tensor from Eq. 22, we first expand the error function, with a Taylor series

$$
S_{j}=-\frac{(1+e) g_{0} \mu m n}{4 \pi(1+\lambda)}\left(I_{n}+I I_{n}\right)
$$

where (for clarity choosing the axes $i$ and $j$ along $x$ and $y$ ):

$$
\begin{aligned}
& I_{n}=\int_{-\infty}^{\infty} \int_{-\infty}^{\infty}\left(\frac{G_{t}}{\sqrt{2 \Theta_{t}}}\right)^{2 n} \exp \left[-\frac{\left(G_{x}-V_{x}\right)^{2}+\left(G_{y}-V_{y}\right)^{2}}{2 \Theta_{t}(1+\lambda)}\right] d G_{x} d G_{y} \\
& I I_{n}=\int_{-\infty}^{\infty} \int_{-\infty}^{\infty} \frac{G_{x}-V_{x}}{\sqrt{2 \Theta_{t}}}\left(\frac{G_{t}}{\sqrt{2 \Theta_{t}}}\right)^{2 n} \exp \left[-\frac{\left(G_{x}-V_{x}\right)^{2}+\left(G_{y}-V_{y}\right)^{2}}{2 \Theta_{t}(1+\lambda)}\right] d G_{x} d G_{y}
\end{aligned}
$$


Here the abbreviations $X=\sqrt{1+\lambda} \cot \theta_{c}, Y=\sqrt{\frac{V_{t}^{2}}{2 \Theta_{t}}} \cot \theta_{c}$ are introduced based on Zhao et al. ${ }^{22}$. Depending on the ratio of $\mathrm{Y}$ over $\mathrm{X}$, the above integrals are carried out differently to the order of $O\left(Y^{2} / \mathrm{X}^{2}\right)$. Firstly, if $Y / X$ is a small quantity, for $\mathrm{n}=0,1,2,3, \ldots$ we have

$$
\begin{aligned}
I_{0} & =\int_{-\infty}^{\infty} \int_{-\infty}^{\infty} \exp \left[-\frac{\left(G_{x}-V_{x}\right)^{2}+\left(G_{y}-V_{y}\right)^{2}}{2 \Theta_{t}(1+\lambda)}\right] d G_{x} d G_{y}=2 \pi \Theta_{t}(1+\lambda) \\
I_{1} & =\int_{-\infty}^{\infty} \int_{-\infty}^{\infty}\left(\frac{G_{t}}{\sqrt{2 \Theta_{t}}}\right)^{2} \exp \left[-\frac{\left(G_{x}-V_{x}\right)^{2}+\left(G_{y}-V_{y}\right)^{2}}{2 \Theta_{t}(1+\lambda)}\right] d G_{x} d G_{y}=\frac{\pi}{2 \Theta_{t}}\left(2 \Theta_{t}(1+\lambda)\right)^{2}\left[1+\frac{V^{2}}{2 \Theta_{t}(1+\lambda)}\right] \\
I_{2} & =\int_{-\infty}^{\infty} \int_{-\infty}^{\infty}\left(\frac{G_{t}}{\sqrt{2 \Theta_{t}}}\right)^{4} \exp \left[-\frac{\left(G_{x}-V_{x}\right)^{2}+\left(G_{y}-V_{y}\right)^{2}}{2 \Theta_{t}(1+\lambda)}\right] d G_{x} d G_{y} \\
& =\frac{\pi}{\left(2 \Theta_{t}\right)^{2}}\left(2 \Theta_{t}(1+\lambda)\right)^{3}\left[2+\frac{V^{2}}{2 \Theta_{t}(1+\lambda)}\left(4+\frac{V^{2}}{2 \Theta_{t}(1+\lambda)}\right)\right] \\
I_{3} & =\int_{-\infty}^{\infty} \int_{-\infty}^{\infty}\left(\frac{G_{t}}{\sqrt{2 \Theta_{t}}}\right)^{6} \exp \left[-\frac{\left(G_{x}-V_{x}\right)^{2}+\left(G_{y}-V_{y}\right)^{2}}{2 \Theta_{t}(1+\lambda)}\right] d G_{x} d G_{y} \\
& =\frac{\pi}{\left(2 \Theta_{t}\right)^{3}}\left(2 \Theta_{t}(1+\lambda)\right)^{4}\left[6+\frac{V^{2}}{2 \Theta_{t}(1+\lambda)}\left(3+\frac{V^{2}}{2 \Theta_{t}(1+\lambda)}\right)\left(6+\frac{V^{2}}{2 \Theta_{t}(1+\lambda)}\right)\right]
\end{aligned}
$$

If the integrals are carried out to the order of $O\left(Y^{2} / X^{2}\right)$, from the above results one can easily find that

$$
I_{n}=\int_{-\infty}^{\infty} \int_{-\infty}^{\infty}\left(\frac{G_{t}}{\sqrt{2 \Theta_{t}}}\right)^{2 n} \exp \left[-\frac{\left(G_{x}-V_{x}\right)^{2}+\left(G_{y}-V_{y}\right)^{2}}{2 \Theta_{t}(1+\lambda)}\right] d G_{x} d G_{y}=2 \pi \Theta_{t}(1+\lambda)^{n+1}\left[1+n \frac{Y^{2}}{X^{2}}\right] n !
$$

While for the second integral carried out to the order of $O\left(Y^{2} / X^{2}\right)$, similarly, using the inductive method, we can find that 


$$
\begin{aligned}
I I_{n} & =\int_{-\infty}^{\infty} \int_{-\infty}^{\infty} \frac{G_{x}-V_{x}}{\sqrt{2 \Theta_{t}}}\left(\frac{G_{t}}{\sqrt{2 \Theta_{t}}}\right)^{2 n} \exp \left[-\frac{\left(G_{x}-V_{x}\right)^{2}+\left(G_{y}-V_{y}\right)^{2}}{2 \Theta_{t}(1+\lambda)}\right] d G_{x} d G_{y} \\
& =\frac{V_{x}}{\sqrt{2 \Theta_{t}}} 2 \pi \Theta_{t}\left[1+\frac{n-1}{2} \frac{Y^{2}}{X^{2}}\right] n ! n(1+\lambda)^{n+1}
\end{aligned}
$$

The total stress can then be found as

$$
\begin{aligned}
S_{j} & =-\frac{(1+e) g_{0} \mu m n}{4 \pi(1+\lambda)} \frac{2}{\sqrt{\pi}} \sum_{n=0}^{\infty} \frac{(-1)^{n} \cot ^{2 n+1} \theta_{c}}{n !(2 n+1)}\left(\frac{V_{x}}{\sqrt{2 \Theta_{t}}} I_{n}+I I_{n}\right) \\
& =-(1+e) g_{0} \mu m n \cot \theta_{c} \frac{\Theta_{t} V_{x}}{\sqrt{2 \pi \Theta_{t}}} A_{1}
\end{aligned}
$$

where $A_{1}=\frac{1}{2\left(1+X^{2}\right)}+\frac{Y^{2}\left(1-X^{2}\right)}{8 X^{2}\left(1+X^{2}\right)^{2}}+\frac{\left(4 X^{2}-Y^{2}\right) \arctan [X]}{8 X^{3}}$

In the opposite limit, if $\frac{Y}{X}=\sqrt{\frac{V^{2}}{2 \Theta_{t}(1+\lambda)}}$ is a large quantity, $\left(G_{t}\right)^{2 n}$ is expanded into a Taylor series around $G_{t}=V$. Then, we find

$$
\begin{aligned}
& I_{n} \approx \pi\left(\frac{V^{2}}{2 \Theta_{t}}\right)^{n} 2 \Theta_{t}(1+\lambda)\left(1+n n \frac{2 \Theta_{t}(1+\lambda)}{V^{2}}\right) \\
& I_{n} \approx \frac{V_{x}}{\sqrt{2 \Theta_{t}}} 2 \pi \Theta_{t}(1+\lambda)\left(\frac{V^{2}}{2 \Theta_{t}}\right)^{n}\left(n \frac{X^{2}}{Y^{2}}+O\left(V^{-4}\right)\right)
\end{aligned}
$$

In this case the total stress can be written as 


$$
\begin{aligned}
S_{j} & =-\frac{(1+e) g_{0} \mu m n}{4 \pi(1+\lambda)} \frac{2}{\sqrt{\pi}} \sum_{n=0}^{\infty} \frac{(-1)^{n} \cot ^{2 n+1} \theta_{c}}{n !(2 n+1)}\left(\frac{V_{x}}{\sqrt{2 \Theta_{t}}} I_{n}+I I_{n}\right) \\
& =-\mu m n g_{0}(1+e) \cot \theta_{c} \frac{\Theta_{t} V_{x}}{\sqrt{2 \pi \Theta_{t}}} A_{2}
\end{aligned}
$$

where $A_{2}=\frac{\sqrt{\pi}}{2 Y} \operatorname{erf}(\mathrm{Y})-\frac{X^{2} \mathrm{e}^{-Y^{2}}\left(2 Y^{2}-1\right)}{4 Y^{2}}-\frac{\sqrt{\pi} X^{2}}{8 Y^{3}} \operatorname{erf}(\mathrm{Y})$

\section{Collisional rate of translation}

For the translational energy dissipation from Eq. 25, we first perform integration with respect to $\mathbf{\Omega}$ and $G_{z}$. Subsequently an expansion is used for the error function

$$
\begin{aligned}
\operatorname{Coll}\left(\frac{1}{2} m c^{2}\right)= & \frac{-2}{\sqrt{\pi}} \sum_{n=0}^{\infty} \frac{(-1)^{n} \cot ^{2 n+1} \theta_{c} \sqrt{2 \Theta_{t}}}{n !(2 n+1)} \int_{-\infty}^{\infty} \int_{-\infty}^{\infty}\left(\frac{G_{t}}{\sqrt{2 \Theta_{t}}}\right)^{2 n+2} \exp \left[-\frac{\left(G_{x}-V_{x}\right)^{2}+\left(G_{y}-V_{y}\right)^{2}}{2(1+\lambda) \Theta_{t}}\right] d G_{x} d G_{y} \\
& -\frac{\sigma}{2}\left(S_{x} \bar{\omega}_{y}-S_{y} \bar{\omega}_{x}\right)
\end{aligned}
$$

In this case,

$$
I I I_{n}=\int_{-\infty}^{\infty} \int_{-\infty}^{\infty}\left(\frac{G_{t}}{\sqrt{2 \Theta_{t}}}\right)^{2 n+2} \exp \left[-\frac{\left(G_{x}-V_{x}\right)^{2}+\left(G_{y}-V_{y}\right)^{2}}{2(1+\lambda) \Theta_{t}}\right] d G_{x} d G_{y}=I_{n+1}
$$

First, when $\frac{Y}{X}=\sqrt{\frac{V^{2}}{2 \Theta_{t}(1+\lambda)}}$ is small, the calculation is carried out to the order of $O\left(\frac{Y^{4}}{X^{4}}\right)$.

According to the calculation of $I_{n}$,

$$
I I I_{n}=2 \pi \Theta_{t}(1+\lambda)^{n+2}\left[1+(n+1) \frac{Y^{2}}{X^{2}}+\frac{n}{4}(n+1) \frac{Y^{4}}{X^{4}}\right](n+1) !=-4 \Theta_{t} \cot \theta_{c}(1+\lambda)^{2} \sqrt{2 \pi \Theta_{t}} B_{1}
$$


where

$$
B_{1}=\frac{2 X^{2}\left(X^{2}+1\right)+Y^{2}\left(X^{2}-Y^{2}+3\right)}{4 X^{2}\left(1+X^{2}\right)^{2}}+\frac{3 Y^{4}\left(1+7 X^{4}\right)}{96 X^{4}\left(1+X^{2}\right)^{3}}+\frac{8 X^{2}\left(2 X^{2}+Y^{2}\right)-Y^{4}}{32 X^{5}} \arctan X
$$

Combining Eq. A6 and A11, the total energy dissipation rate is,

$$
\begin{aligned}
\operatorname{Coll}\left(\frac{1}{2} m c^{2}\right)= & n m g_{0} \Theta_{t} \sqrt{\frac{\Theta_{t}}{2 \pi}}\left\{-\left(1-e^{2}\right)+\mu^{2}(1+e)^{2}\left(1-\frac{e^{-\frac{Y^{2}}{1+X^{2}}}}{1+X^{2}}\right)\right\} \\
& +\mu n m g_{0}(1+e) \Theta_{t} \cot \theta_{c}\left\{(1+\lambda) \sqrt{\frac{2 \Theta_{t}}{\pi}} B_{1}+\frac{\sigma}{2} \frac{\left(V_{x} \bar{\omega}_{y}-V_{y} \bar{\omega}_{x}\right)}{\sqrt{2 \pi \Theta_{t}}} A_{1}\right\}
\end{aligned}
$$

Second, when $\frac{Y}{X}=\sqrt{\frac{V^{2}}{2 \Theta_{t}(1+\lambda)}}$ is large, $\left(G_{t}\right)^{2 n}$ is expanded into a Taylor series around $G_{t}=V$.

Then we have

$$
\begin{aligned}
I I I_{n} & =2 \pi \Theta_{t}(1+\lambda)\left(\frac{V^{2}}{2 \Theta_{t}}\right)^{n+1}\left(1+(n+1)^{2} \frac{2 \Theta_{t}(1+\lambda)}{V^{2}}+\frac{n^{2}}{2}(n+1)^{2}\left(\frac{2 \Theta_{t}(1+\lambda)}{V^{2}}\right)^{2}\right) \\
& =-4 \pi \Theta_{t}(1+\lambda) \cot \theta_{c} \sqrt{\frac{2 \Theta_{t}}{\pi}} \frac{V^{2}}{2 \Theta_{t}} B_{2}
\end{aligned}
$$

where

$$
B_{2}=\frac{\sqrt{\pi}}{2 Y} \operatorname{erf}(Y)+\frac{X^{2} \sqrt{\pi}}{8 Y^{3}} \operatorname{erf}(Y)+\frac{3-2 Y^{2}}{4 Y^{2}} X^{2} \mathrm{e}^{-Y^{2}}+\frac{\sqrt{\pi} X^{4}}{64 Y^{5}} \operatorname{erf}(Y)-\frac{1+22 Y^{2}-36 Y^{4}+8 Y^{6}}{32 Y^{4}} X^{4} \mathrm{e}^{-Y^{2}}
$$

Fig. A1 shows that the last term in the above equation can be neglected when the magnitude of the normalized slip velocity is larger than unity because the first two terms are dominate. Because this last term can cause oscillations for small values of the normalized slip velocity, we drop this term. This is also permissible because in this second case $Y / X$ is assumed to be large. 
Combining Eq. A9 and A11, the total energy dissipation rate is,

$$
\begin{aligned}
\operatorname{Coll}\left(\frac{1}{2} m c^{2}\right)= & n m g_{0} \Theta_{t} \sqrt{\frac{\Theta_{t}}{2 \pi}}\left\{-\left(1-e^{2}\right)+\mu^{2}(1+e)^{2}\left(1-\frac{e^{-\frac{Y^{2}}{1+X^{2}}}}{1+X^{2}}\right)\right\} \\
& +\mu n m g_{0}(1+e) \Theta_{t} \cot \theta_{c}\left\{\sqrt{\frac{2 \Theta_{t}}{\pi}} \frac{V^{2}}{2 \Theta_{t}} B_{2}+\frac{\sigma\left(V_{x} \bar{\omega}_{y}-V_{y} \bar{\omega}_{x}\right)}{2 \sqrt{2 \pi \Theta_{t}}} A_{2}\right\}
\end{aligned}
$$

Calculation of the rotational energy dissipation rate

Similarly, after Taylor expansion of the error function and integrating over $\boldsymbol{\Omega}$ and $G_{z}$, the rotational energy dissipation becomes

$$
\begin{aligned}
& -D_{r}=\frac{5 n m g_{0}}{2} \mu^{2}(1+e)^{2} \Theta_{t} \sqrt{\frac{\Theta_{t}}{2 \pi}}\left(1-\frac{e^{-\frac{V^{2} \cot ^{2} \theta_{c}}{2 \Theta_{t}\left(1+(1+\lambda) \cot ^{2} \theta_{c}\right)}}}{1+(1+\lambda) \cot ^{2} \theta_{c}}\right) \\
& -\frac{n m \sigma g_{0}}{2} \frac{\mu(1+e)}{4 \pi(1+\lambda)} \int_{-\infty}^{\infty} \int_{-\infty}^{\infty} \frac{G_{x} \bar{\omega}_{y}-G_{y} \bar{\omega}_{x}}{G_{t}} \operatorname{erf}\left(\frac{G_{t} \cot \theta_{c}}{\sqrt{2 \Theta_{t}}}\right) \exp \left[-\frac{\left(G_{x}-V_{x}\right)^{2}+\left(G_{y}-V_{y}\right)^{2}}{2(1+\lambda) \Theta_{t}}\right] d G_{x} d G_{y}
\end{aligned}
$$

In a very similar fashion to the calculation of the shear stress tensor, according to Eq. A6 and A9, we can easily find that

$$
\begin{aligned}
\operatorname{Coll}\left(\frac{1}{2} I \omega^{2}\right)= & \frac{5 n m g_{0}}{2} \mu^{2}(1+e)^{2} \Theta_{t} \sqrt{\frac{\Theta_{t}}{2 \pi}}\left(1-\frac{e^{-\frac{Y^{2}}{1+X^{2}}}}{1+X^{2}}\right) \\
& -\frac{\sigma}{2} g_{0} \mu n m(1+e) \Theta_{t} \cot \theta_{c} \frac{\left(V_{x} \bar{\omega}_{y}-V_{y} \bar{\omega}_{x}\right)}{\sqrt{2 \pi \Theta_{t}}}\left\{\begin{array}{l}
A_{1}, \text { if } Y / X \text { is small } \\
A_{2}, \text { if } Y / X \text { is large }
\end{array}\right.
\end{aligned}
$$

